\title{
Efecto antihelmíntico in vitro de extractos vegetales en nematodos gastrointestinales de ovinos de pelo
}

\author{
José T. López C. ${ }^{1}$ - Roberto G. Garduño ${ }^{2}$ - Glafiro Torres H. ${ }^{3}$ - \\ Silvio Gutiérrez C..$^{a}$ - Victorino Gómez $V^{b}$ - Francisco Reyes $M .^{c}$ \\ Universidad Autónoma Chapingo, México
}

Recepción: $12 / 07 / 2015$

Aceptación: 3/08/2015

\section{Resumen}

Debido al incremento de resistencia de los nematodos gastrointestinales (NGI) a los antihelmínticos, se propuso como objetivo evaluar in vitro la eficacia de extractos de diferentes plantas. Se formularon extractos hidroalcohólicos que se aplicaron sobre huevos y larvas infectantes (L3) de NGI. En el caso de las larvas se realizaron dos pruebas: en la primera se usó extracto de guaje (L. diversifolia), conacaste negro (E. cyclocarpum) y pega-pega (D. tortuosum); y para el segundo bioensayo se utilizó el extracto de guaje (L. leucocephala), mangle (R. mangle) y neem (A. indica). En la prueba de inhibición de la eclosión de huevos (IEH) se usaron hojas de neem (A. indica), de hierba santa (P. auritum) y de hierba del zorrillo (P. alliacea).

Los huevos y larvas se obtuvieron de ovinos infectados de manera natural en pastoreo. Las larvas infectantes se desenvainaron con hipoclorito de sodio (0.187\%), después se agregaron 100 larvas por pozo en agua destilada y se desafiaron con los extractos a través de una prueba in vitro de motilidad larval con observación directa. Los resultados mostraron que los extractos de L. leucocephala y A. indica presentaron un grado de acción larvicida en NGI similar a levamisol. Con el extracto de L. leucocephala se obtuvo la menor dosis letal (DL50=0.045mg/ml-1) contra L3. En la eclosión de huevos, los mejores resultados se obtuvieron con el extracto de P. alliacea y P. auritum

Palabras clave: nematodos, resistencia antihelmíntica, metabolitos secundarios, extractos, PROBIT.

\begin{abstract}
Due to the growth of resistance of the gastrointestinal nematodes (GIN) to the anthelmintic ones, it was proposed as a goal to evaluate in vitro the efficacy of essences from different plants. There were formulated hydro-alcoholic essences that were put on over eggs and infective larvae (L3) of GIN. In the case of the larvae, there were carried two tests: in the first one, there were used gourd essence (L. diversifolia), black Elephant's ear (E.cyclocarpum) and pega-pega (D. tortuosum); whereas for the second bioassay, there were used gourd essence (L. Leucocephala), mangrove (R. mangle) and neem (A. indica). In the inhibition test of the hatching of eggs (ITHE), there were used neem leaves (A. indica), yerba santa leaves (P. auritum) and skunk weed leaves (P. alliacea).

Eggs and larvae were obtained from infected ovine in a natural way while grazing. The infective larvae were unsheathed with sodium hypochlorite $(0.187 \%)$. After, there were added 100 larvae per pit on distilled water and they challenged themselves with their essence through an in vitro test of larval motility with direct observation. Treatments were the essences at different concentrations $(100,50,25,12.5,6.25,3.13,1.56,0.78,0.39,0.20,0.10,0.05 \mathrm{mg} / \mathrm{ml}-1)$. The levamisole on its commercial and diluted presentation $(120,60,30,15,7.5,3.75,1.88,0.94,0.47,0.23,0.12$, and $0.06 \mathrm{mg} / \mathrm{ml}-1)$ was used as a reference to determine the effectiveness of the essences and the larvae in distilled water to determine the natural mortality. In the ITHE test, there were also used the same concentrations. Data were analyzed through the process PROBIT. The results demonstrated that the essences of L. leucocephala and A. indica presented a stage of larvicidal action in GIN similar to levamisole. With the essence of $\mathrm{L}$. leucocephala, there was obtained the least lethal dose (DL50 $=0.045 \mathrm{mg} / \mathrm{ml}-1)$ against L3. In the hatching of eggs, the best results were obtained with the essence of P. alliance and P. auritum.
\end{abstract}

Key words: nematodes, anthelmintic resistance, secondary metabolites, essences, PROBIT

1,a,b,c Unidad Regional Universitaria Sursureste. Universidad Autónoma Chapingo, km 7.5 Carretera Teapa-Vicente Guerrero, Teapa, Tabasco, México.

2. Autor de correspondencia: Roberto González Garduño. E-mail: robgardu@hotmail.com

3. Colegio de Posgraduados. Instituto de Recursos Genéticos y Productividad. Ganadería. Campus Montecillos. Montecillos, Estado de México. CP 53230. 


\section{Introducción}

El uso frecuente de los antihelmínticos para el control de los parásitos ha sido una de las principales causas que ha provocado el desarrollo de resistencia en las principales especies de nematodos gastrointestinales (NGI), por lo que existen reportes en muchas partes del mundo (Papadopoulos, 2008; Shalaby, 2013); y actualmente se demuestra la presencia de resistencia hacia los principales ingredientes activos usados como desparasitantes (Taylor et al., 2009). Debido al aumento de casos de resistencia en ovinos, bovinos, cabras y caballos, es más frecuente observar una baja efectividad de los desparasitantes, lo que ha originado preocupación e iniciado la búsqueda de otros principios activos, por lo que el estudio de plantas con potencial antiparasitario se ha convertido en un tema de interés internacional (Githiori et al., 2006; Marie-Magdeleine et al., 2010); ya que la tendencia es obtener alimentos inocuos y evitar la contaminación del ambiente con residuos de productos químicos sintéticos (Athanasiadou et al., 2001).

Diversos estudios confirman que algunas plantas han sido utilizadas para el control de nematodos gastrointestinales en diferentes partes del mundo. Se han estudiado hojas, tallos, frutos y semillas buscando metabolitos que tengan acción antihelmíntica. Destacan los estudios de leguminosas como el quebracho (Schinopsis sp.) (Hoste et al., 2006),
Acacia y Leucaena (Makkar et al., 2007), Lysiloma y Havardia albicans (Brunet et al., 2008; Alonso-Díaz et al., 2010), en cuyas plantas se indica la presencia de compuestos como taninos y otros metabolitos secundarios con acción antihelmíntica y por lo tanto con posibilidades para su uso en el control de NGI.

En países con clima cálido como Cuba, existe experiencia con el uso de fitofármacos en el control parasitario. Una de las plantas que mejores resultados ha dado es la piña de ratón (Bromelia pinguin), la cual ha sido efectiva contra Haemonchus spp (Marrero et al., 1994); y en México se empleó contra Oesophagostomun columbianum (Olivares, 2001). En Yucatán, se han identificado plantas ricas en taninos (Lisiloma latisiliquum, $\mathrm{Ha}$ vardia albican), que afectan las fases adultas y larvarias de $\mathrm{H}$. contortus, ya que reducen la longitud y la prolificidad del parásito (Torres-Acosta et al., 2012).

De las técnicas para determinar la actividad de los extractos de plantas, las pruebas in vitro han sido ampliamente usadas, entre ellas, la de inhibición de la eclosión de huevos (IEH), la de desarrollo de la migración larvaria y la de inhibición de la motilidad de parásitos adultos (Marie-Magdeleine et al., 2009). Los test in vitro son un medio apropiado para evaluar extractos de plantas debido a que son rápidos de realizar y económicos, comparados a los test in vivo. Por ello, el presente estu- 
dio se llevó a cabo la evaluación del potencial antiparasitario de extractos de plantas mediante la prueba de inhibición de la eclosión de huevos y el de motilidad de larvas en tercer estadio de nematodos gastrointestinales de ovinos infectados naturalmente.

\section{Materiales y métodos}

El experimento se llevó a cabo en el laboratorio de sanidad animal de la Unidad Regional Universitaria Sursureste (URUSSE), de la Universidad Autónoma Chapingo (UACh), ubicada en San José Puyacatengo, del Municipio de Teapa, Tabasco; que se encuentra a una altitud de $60 \mathrm{msnm}$ y con coordenadas $17^{\circ} 34^{\prime}$ 30 " latitud Norte y $92^{\circ} 56^{\prime} 15^{\prime \prime}$ longitud Oeste. El clima de la región es Af(m)wi' de acuerdo a las modificaciones climáticas de Köppen, es decir, cálido húmedo con lluvias todo el año. La temperatura promedio anual es $25.8^{\circ} \mathrm{C}$ y la precipitación de 3,975.5mm (García, 1988).

Se utilizaron los extractos de ocho plantas en las cuales se utilizaron diferentes partes de ellas. Se usaron las hojas de neem (A. indica), hierba santa (P. auritum), y hierba del zorrillo (P. alliacea); la planta completa de pega-pega (D.tortuosum), y la corteza de mangle (R. mangle), guaje (L. leucocephala subsp. Glabrata), oreja de ratón o conacaste negro (E. cyclocarpum) y guaje de montaña (L. diversifolia).
El material colectado se deshidrató a la sombra, a temperatura ambiente durante cuatro a siete días, hasta lograr un contenido de 8 a $10 \%$ de humedad determinada por la reducción del peso del material verde al peso en seco. Después se obtuvo la harina con un molino manual para granos (marca Estrella), obteniendo partículas de 1 a $2 \mathrm{~mm}$, y se almacenó en recipientes de plástico como lo recomienda Álvarez et al. (1989).

De la harina de cada planta se preparó una solución al $10 \% \mathrm{P} / \mathrm{V}$, en la cual se mezclaron $10 \mathrm{~g}$ de polvo de planta en $100 \mathrm{ml}$ de una solución hidroalcohólica compuesta por $80 \mathrm{ml}$ de etanol (C2H6O) al 70\% y 20ml de agua destilada. La extracción se hizo mediante remojo y agitación durante $15 \mathrm{~min}$, y luego un reposo de 48 horas a temperatura ambiente, a la sombra en un lugar fresco. A las 24 horas se agitó el frasco nuevamente para asegurar la correcta homogenización del material vegetal con la solución hidroalcohólica. Los extractos obtenidos se filtraron en un tamiz del número 100 (0.149mm de apertura) para separar las partículas vegetales de mayor tamaño, seguida de una centrifugación a 2500rpm durante cinco minutos para sedimentar y eliminar las partículas más pequeñas. El extracto resultante se envasó en frascos de plástico de $250 \mathrm{ml}$ y, posteriormente, se utilizó en la prueba in vitro de acuerdo a la metodología descrita posteriormente. 


\section{Prueba de motilidad larval}

Para los bioensayos se tomaron muestras de heces de ovinos de la cruza Katahdin x Pelibuey infectados de manera natural en pastoreo, en el módulo de producción de ovinos de la UACh. Se realizó la determinación de conteo fecal de huevos de nematodos por gramo de heces (hpg) mediante la técnica de McMaster (Thienpont et al., 1986) la cual es una técnica de flotación en la que se colocan dos gramos de heces en $28 \mathrm{ml}$ de solución salina saturada, se llena la cámara de McMaster y se contabilizan los huevos de nematodos en la cuadrícula y los resultados se multiplican por 50. Se seleccionaron como donadores aquellos animales que tuvieron conteos fecales mayores a 1000hpg. Con las heces positivas a huevos de nematodos se realizaron coprocultivos que consistieron en colocar $20 \mathrm{~g}$ de heces en un recipiente de plástico tapado con gasa; cada tercer día se le agregaron tres mililitros de agua. Los cultivos se dejaron siete días a temperatura ambiente; después se recuperaron las larvas mediante la técnica de Baermann que consistió en colocar las heces en una gasa, la cual se colocó en un embudo con agua al que se adhirió una manguera de latex y un tubo de ensayo. Una vez colectadas se limpiaron mediante gradientes de densidad con sacarosa al 40\%, de acuerdo a la metodología descrita por Thienpont et al. (1986) y en la que las larvas se recuperan en la interfase de la sacarosa y el agua, después de una centrifugación de 5 minutos a 2500 revoluciones por minuto (rpm).

Para los bioensayos, las larvas se desenvainaron con hipoclorito de sodio al $0.187 \%$, para ello se centrifugaron las larvas en tubos eppendorf de $15 \mathrm{~cm}$, a $2500 \mathrm{rpm}$ durante $5 \mathrm{mi}-$ nutos. Se eliminó el agua con una pipeta hasta dejar $1 \mathrm{ml}$ y se re-suspendieron las larvas con agua destilada. Esto se realizó tres veces para eliminar el exceso de cloro y, posteriormente, se cuantificaron mediante la determinación de 10 alícuotas de 10 microlitros $(\mu \mathrm{L})$.

La prueba in vitro se realizó en dos etapas: en la etapa I se usó la técnica de migración larval de acuerdo a lo descrito por Wagland et al. (1992), con observación directa de la motilidad de las larvas. Se realizaron los bioensayo en dos ocasiones, en el primero se evaluó el efecto del extracto de guaje (L. diversifolia), conacaste negro (E. cyclocarpum) y pega-pega (D. tortuosum); y para el segundo bioensayo se utilizó el extracto de guaje (L. leucocepha$\underline{\text { la) }}$, mangle (R. mangle) y neem (A. indica). En ambas pruebas se determinó también la motilidad de las larvas en agua destilada para conocer el porcentaje de mortalidad natural; también se determinó la motilidad de las larvas usando levamisol (Ripercol L 12\%, Pfizer, $7.5 \mathrm{mg} / \mathrm{kg}-1$ ) para determinar la efectividad de un producto químico y tener una referencia de comparación. Para la determinación de 
las categorías de las larvas se tomaron como criterios: larvas muertas, aquellas que se observaron rectas y sin movimiento; mientras que las larvas vivas fueron aquellas que estuvieran enrolladas o bien en movimiento.

Las evaluaciones de los extractos se realizaron por duplicado en cajas de poliestireno de 96 pozos, en los cuales se colocaron aproximadamente 100L3 por pozo en $100 \mu \mathrm{L}$ de agua, posteriormente sólo en la primera fila se agregaron $100 \mu \mathrm{L}$ de cada uno de los extractos vegetales, y también de agua destilada $y$ de levamisol (volumen total de $200 \mu \mathrm{L}$ ). De la primera columna se tomaron $100 \mu \mathrm{L}$ y se agregaron a la columna dos; y así sucesivamente, con lo que se consiguieron diluciones dobles en las doce columnas de la placa. Las concentraciones de los extractos, por lo tanto fueron: 100, 50, 25, 12.5, 6.25, 3.13, 1.56, 0.78, $0.39,0.20,0.10$ y $0.05 \mathrm{mg} / \mathrm{ml}-1$. El levamisol al $12 \%$ en su presentación comercial se usó como referencia para comparar los resultados de los extractos, y constituyó un control negativo que se evaluó también en diluciones dobles $(120,60,30,15,7.5,3.75,1.88,0.94,0.47$, $0.23,0.12$ y $0.06 \mathrm{mg} / \mathrm{ml}-1)$. También se colocaron larvas en agua destilada para determinar la mortalidad natural (control positivo). La placa se tapó con papel encerado y se incubó durante 24 horas a temperatura ambiente $\left(26^{\circ} \mathrm{C}\right)$. La lectura de la movilidad de las larvas se realizó a las 24 horas post-interacción.

\section{Prueba de inhibición de la eclosión de huevos}

El ensayo se realizó a partir de las modificaciones a las técnicas descritas por Marie-Magdeleine et al., (2010) y Arece et al., (2012). Se evaluaron por duplicado los extractos de tres plantas. Se usaron hojas de neem (A. indica), de hierba santa (P. auritum) y de hierba del zorrillo (P. alliacea), en ocho concentraciones $(100,50,25,12.5,6.25,3.12,1.56$ y $0.78 \mathrm{mg} /$ ml-1), el levamisol al 12\% como referencia de comparación con los extractos $(120,60,30$, $15,7.5,3.75,1.88$ y $0.94 \mathrm{mg} / \mathrm{ml}-1)$ y los huevos de NGI en agua destilada para determinar la mortalidad natural.

Se colectaron huevos de nematodos que se obtuvieron mediante flotación con una solución salina de $\mathrm{NaCl}$. La muestra se centrifugó en tubos eppendorf de $15 \mathrm{ml}$ a 2500rpm durante cinco minutos, y se desechó el material precipitado. Se limpió la muestra en un tamiz de $400(0.038 \mathrm{~mm}$ de apertura) con agua corriente, y los huevos recuperados en el tamiz se colocaron en una solución de sacarosa al $40 \%$; y nuevamente se centrifugaron en las mismas condiciones. Posteriormente se recuperaron los huevos de la interfase y se filtraron en el tamiz del número 400, quedando los huevos en la superficie del tamiz. Los huevos se suspendieron en agua destilada en tubos eppendorf de $15 \mathrm{ml}$ y se homogenizaron para su cuantificación mediante el conteo de 10 alícuotas de $10 \mu \mathrm{L}$, y se distribuyeron $100 \mu \mathrm{L}$ 
con 100 huevos por pozo en la placa de 96 pozos. Luego se agregaron $100 \mu \mathrm{L}$ de cada extracto a una concentración inicial de 100mg/ $\mathrm{ml}-1$; de igual forma el levamisol con 120mg/ ml-1 de concentración inicial. Posteriormente, se distribuyeron $100 \mu \mathrm{L}$ de cada tratamiento entre los pozos en diluciones dobles; consecutivamente se dejó incubar por 12 horas. Transcurrido ese tiempo, se detuvo la eclosión con $10 \mu \mathrm{L}$ de solución de Yodo lugol. De cada pozo se tomaron cinco alícuotas $(20 \mu \mathrm{L})$ y se hizo el conteo de los huevos que quedaron sin eclosionar, y del número de larvas con lo que se calculó el porcentaje de eclosión.

\section{Análisis estadístico}

Para el análisis de la motilidad larval y para la eclosión de huevos, se calcularon los porcentajes de efectividad (mortalidad larvaria y eclosión) para cada extracto mediante un análisis Probit (DL50) con SAS (SAS, 1999), considerando el siguiente modelo:

$$
\begin{gathered}
\operatorname{Pr}(\text { Respuesta })=\mathrm{C}+(1-\mathrm{C}) \mathrm{F}\left(\mathrm{x}^{\prime} \beta\right)= \\
\mathrm{C}+(1-\mathrm{C}) \Phi(\mathrm{b} 0+\mathrm{b} 1 \mathrm{x} \log 10(\text { Dosis }))
\end{gathered}
$$

Dónde:

$\boldsymbol{\beta}$ es un vector de parámetros estimados

F es una función de distribución acumulativa (Normal)

$\mathbf{X}$ es un vector de variables explicativas

Pr es la probabilidad de una respuesta

C es la tasa de respuesta natural (proporción de individuos que responden a la dosis cero).

\section{Resultados y discusión}

En la prueba de motilidad larval, el extracto de L. diversifolia, D. tortuosum, L. leucocephala y A. indica $(6.68,7.51,0.045$ y 2.31 $\mathrm{mg} / \mathrm{ml}-1$ ) causaron un aumento significativo en la mortalidad de las larvas conforme se elevó la concentración del producto $(\mathrm{P}<0.01)$; y la DL50 fue similar a la observada con levamisol (5.54 y $2.14 \mathrm{mg} / \mathrm{ml}-1)$. En lo referente al número de individuos que responden a la dosis cero (agua destilada), en las ecuaciones PROBIT se observaron tasas de mortalidad natural entre 3 a 7\% (C); y para el resto de las larvas (1-C), la pendiente de la regresión (b1) fue positiva en todos los casos - excepto para R. mangle y E. ciclocarpum - lo que indica que no existió una relación directa entre la concentración y la mortalidad larvaria en estos dos extractos (ver tabla 1). 
Tabla 1. Ecuaciones Probit, coeficientes de regresión y concentración letal de extractos vegetales contra larvas infectantes de nematodos gastrointestinales

\begin{tabular}{|c|c|c|c|c|c|}
\hline Antihelmíntico & Ecuación PROBIT & $\mathbf{b}_{0}$ & $b_{1}$ & $\mathrm{CL}_{50}$ & $\mathrm{DL}_{50}$ \\
\hline \multicolumn{6}{|c|}{ Primera prueba in vitro } \\
\hline L. diversifolia & $\operatorname{Pr}=0.05+0.95(\Phi(-2.54+3.09 \times \log 10($ Dosis $)))$ & ** & $* *$ & 0.83 & 6.68 \\
\hline E. cyclocarpum & $\operatorname{Pr}=0.03+0.97(\Phi(-39.67+27.14 \times \log 10($ Dosis $)))$ & ns & ns & 1.46 & 28.94 \\
\hline D. tortuosum & $\operatorname{Pr}=0.04+0.96(\Phi(-3.57+4.08 \times \log 10($ Dosis $)))$ & $* *$ & $* *$ & 0.88 & 7.51 \\
\hline Levamisol al $12 \%$ & $\operatorname{Pr}=0.06+0.94(\Phi(-1.20+1.62 \times \log 10($ Dosis $)))$ & $* *$ & $* *$ & 0.74 & 5.54 \\
\hline \multicolumn{6}{|c|}{ Segunda prueba in vitro } \\
\hline L. leucocephala & $\operatorname{Pr}=0.03+0.97(\Phi(0.61+0.45 \times \log 10($ Dosis $)))$ & ** & ** & -1.34 & 0.045 \\
\hline R. mangle & $\operatorname{Pr}=0.07+0.93(\Phi(-23.10+26.9 \times \log 10($ Dosis $)))$ & ns & ns & 0.86 & 7.23 \\
\hline A. indica & $\operatorname{Pr}=0.04+0.96(\Phi(-0.64+1.75 \times \log 10($ Dosis $)))$ & * & $* *$ & 0.36 & 2.31 \\
\hline Levamisol al 12\% & $\operatorname{Pr}=0.04+0.96(\Phi(-0.46+1.31 \times \log 10($ Dosis $)))$ & $* *$ & $* *$ & 0.35 & 2.14 \\
\hline
\end{tabular}

Nota: $\mathbf{b}_{\mathbf{0}}$ : Ordenada al origen, $\mathrm{b}_{1}$ : pendiente de regresión, $\mathbf{C L}_{50}$ : Concentración letal media, $\mathbf{D L}_{50}$ : Dosis letal media $(\mathrm{mg} / \mathrm{ml}-1)$. ns: no significativo $(\mathrm{P}>0.05),{ }^{\star}$ Significativo $(\mathrm{P}<0.05),{ }^{\star *}$ altamente significativo $(\mathrm{P}<0.01)$.

En el primer experimento, el levamisol tuvo una $\mathrm{DL}_{50}$ de $5.54 \mathrm{mg} / \mathrm{mL}^{-1}$. Sin embargo, para lograr un control del 99\% de las larvas $\left(\mathrm{DL}_{99}\right)$ se obtuvo una concentración de $150.9 \mathrm{mg} /$ $\mathrm{ml}^{-1}$, lo que indica problemas de resistencia antihelmíntica debido a que se requiere una alta concentración para lograr una alta mortalidad. Esta situación ya se ha confirmado en otro estudio con una prueba en campo, utilizando este mismo producto, el cual posee una efectividad de tan solo 30\% (González-Garduño et al., 2014); por lo que los resultados de los extractos resultan alentadores en el control de nematodos, y sobre todo cuando las dosis son menores a las de los antihelmínticos comerciales, como ocurrió con el extracto de D. tortuosum $(28 \mathrm{mg} / \mathrm{mL}-1)$ y el de L. diver- sifolia, que con menores concentraciones $\left(38 \mathrm{mg} / \mathrm{mL}^{-1}\right)$ lograron el $99 \%$ de mortalidad en las larvas (figura 2).

En L. diversifolia existen escasos estudios sobre la acción nematicida de este producto; y los resultados muestran que posee acción ovicida en Haemonchus contortus de sólo $33 \%$ a una dosis de $1.25 \mathrm{mg} / \mathrm{mL}^{-1}$ (Florence y Mbida, 2011). Para el caso de D. tortuosum y E. cyclocarpum no se encontraron referencias sobre su efecto en nematodos gastrointestinales, pero si existen estudios en nematodos que afectan a los cultivos como Meleidogine (Quesenberry y Dunn, 1987). 


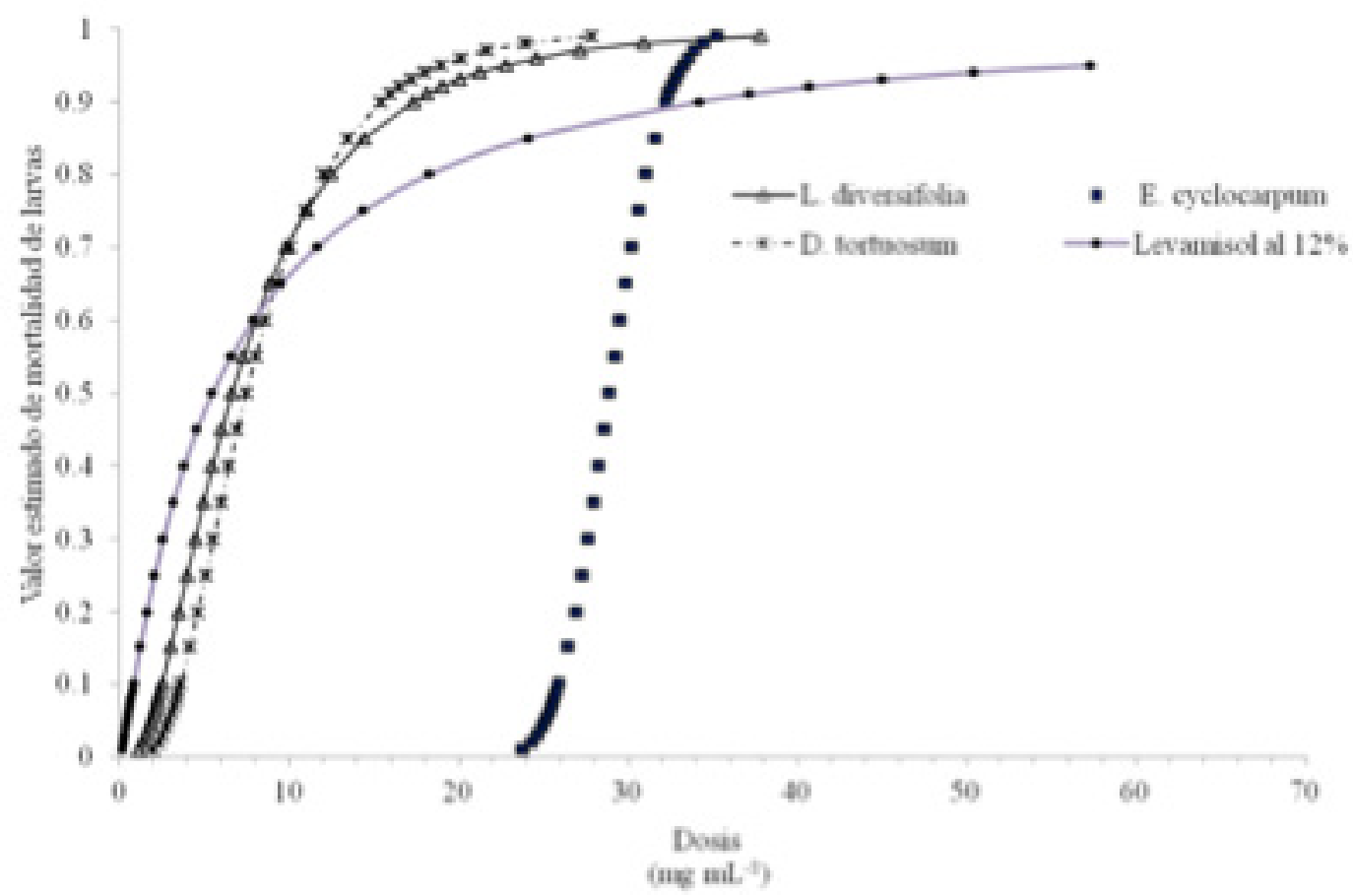

Figura 1. Resultado comparativo de la mortalidad de larvas infectantes en la prueba in vitro utilizando extractos de L. diversifolia, E. cyclocarpum, D. tortuosum y levamisol al 12\%.

En el segundo ensayo, el levamisol tuvo una $\mathrm{DL}_{50}$ más baja $\left(2.14 \mathrm{mg} / \mathrm{mL}^{-1}\right)$ que en el primer ensayo (figura 2). Sin embargo, las concentraciones usadas demuestran un bajo efecto nematicida. Esto se atribuyó al uso frecuente de este antihelmíntico durante un periodo de cuatro años en el rebaño del cual se obtuvieron las larvas. Esta baja eficacia ya se había indicado previamente en un estudio del mismo rebaño, en el cual se utilizaron tres antihelmínticos (levamisol, ivermectina y albendazol + closantel), con la intención de reducir el número de huevos de NGI en ovinos en pastoreo (González-Garduño et al., 2010).

El extracto de corteza de L. leucocephala fue el producto que tuvo la dosis letal más pequeña $\left(0.045 \mathrm{mg} / \mathrm{mL}^{-1}\right)$, lo cual demuestra la efectivi- dad en contra de larvas de NGI. Alonzo-Díaz et al. (2010) indican que los taninos de esta planta actúan como agentes antihelmínticos naturales en los rumiantes. En estudios realizados en Yucatán (UADY) en H. contortus, observaron que con una concentración de $2400 \mu \mathrm{g} / \mathrm{mL}^{-1}$ del extracto de las hojas de esta planta se afectó la migración del $43.5 \pm$ $3.7 \%$ de larvas infectantes de la cepa UNAM; el $73.4 \pm 6.0 \%$ de la cepa CENID-INIFAP y el $58.2 \pm 4.3 \%$ de la cepa UADY (CalderonQuintal et al., 2010). También Medina y Sánchez (2006) indicaron que, utilizando follaje de Leucaena en la suplementación de ovinos, existe una mejor respuesta animal en la ganancia de peso y disminución de huevos de estrongílidos. 


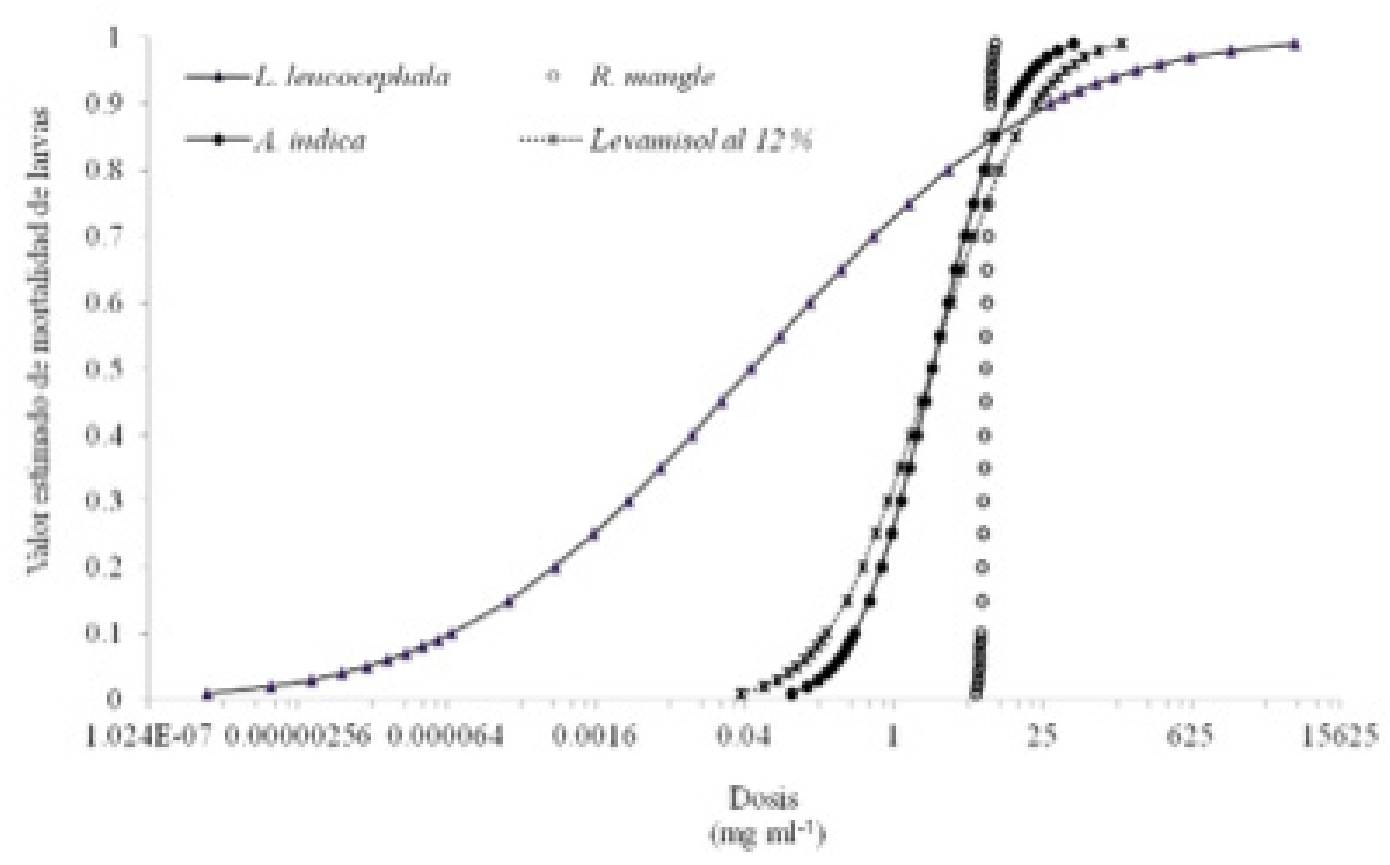

Figura 2. Resultado comparativo de la mortalidad de larvas infectantes en la prueba in vitro utilizando extractos de L. leucocephala, R. mangle, A. indica y levamisol.

Los resultados obtenidos en $\mathrm{R}$. mangle difieren a lo indicado en Cuba para el extracto acuoso de la corteza del mangle rojo, el cual a una concentración de $50 \mathrm{mg} / \mathrm{mL}^{-1}$ alcanzó un $72 \%$ de efectividad y $68.4 \%$ para el extracto acuoso residual en la mortalidad de Haemonchus spp. y Trichostrongylus spp. (Aleman et al., 2011). Esta efectividad posiblemente esté relacionada con las diferencias en la estructura química, grado de proliferación de los metabolitos secundarios y la concentración de los diferentes polifenoles en las especies, posiblemente también las condiciones de temperatura y época del año pudieran originar estas diferencias (Reed, 1995).
Con el extracto de las hojas del neem (A. indica) se obtuvo una $\mathrm{DL}_{50}$ de $2.31 \mathrm{mg} / \mathrm{mL}^{-1}$. El efecto antihelmíntico del extracto del neem fue comparable con la acción larvicida del levamisol (figura 3). Los resultados muestran algunas propiedades anti-parasitarias, lo cual coincide con lo expresado por otros autores en varios experimentos in vitro (Pietrosemoli et al., 1999; Salazar, 2004; Devon-Ronald et al., 2012), en los cuales se demuestra que la administración - cada 48 horas - con una dosis de $0.67 \mathrm{~g} / \mathrm{Kg}^{-1}$, del extracto acuoso de hojas de neem, durante tres días, logra controlar los géneros Strongyloides, Haemonchus, Ostertagia y Trichostrongylus en un 100\%. Pero son necesarias varias aplicaciones seguidas 
para mantener la acción antiparasitaria por efecto acumulativo, ya que la cantidad de principio activo en cada tratamiento es baja en comparación con las de síntesis química; una tendencia general en los fitofármacos.

\section{Prueba de reducción de la eclosión de huevos}

Todos los extractos utilizados tuvieron un efecto importante al inhibir la eclosión de huevos de nematodos. La pendiente de la regresión fue significativa $(\mathrm{P}<0.05)$ en todos los casos excepto con levamisol. Esto significa que conforme se incrementó la dosis del extracto, se redujo la tasa de eclosión de los huevos (tabla 2). En el caso del levamisol no hubo una relación directa entre la cantidad de huevos eclosionados y las concentraciones del producto, lo cual demuestra, que este fármaco carece de acción ovicida (Lanusse y Prichard, 1993; Márquez-Lara, 2003).

Tabla 2. Ecuaciones Probit, coeficientes de regresión y concentración letal de los extractos de tres plantas utilizados en bioensayos de eclosión de huevos de nematodos gastrointestinales

\begin{tabular}{|l|l|l|l|l|c|}
\hline \multicolumn{1}{|c|}{ Antihelmíntico } & Ecuación PROBIT & $\mathbf{b}_{\mathbf{0}}$ & $\mathbf{b}_{\mathbf{1}}$ & $\mathbf{C L}_{50}$ & $\mathbf{D L}_{50}$ \\
\hline A. indica & $\operatorname{Pr}=0.34+0.66(\Phi(-2.14+1.87 \times \log 10($ Dosis $)))$ & $* *$ & $\star *$ & 1.15 & 14 \\
\hline P. autiritum & $\operatorname{Pr}=0.36+0.64(\Phi(-1.83+1.94 \times \log 10($ Dosis $)))$ & $\star$ & $* *$ & 0.94 & 8.72 \\
\hline P. alliacea & $\operatorname{Pr}=0.34+0.66(\Phi(-2.19+2.77 \times \log 10($ Dosis $)))$ & $\star$ & $\star *$ & 0.79 & 6.17 \\
\hline Levamisol & $\operatorname{Pr}=0.38+0.62(\Phi(-0.08+0.34 \times \log 10($ Dosis $)))$ & ns & ns & 0.25 & 1.79 \\
\hline
\end{tabular}

Nota: $\mathbf{b}_{0}$ : Ordenada al origen. $\mathbf{b}_{1}$ : Pendiente de la regresión. $\mathbf{C L}_{50}$ : Concentración letal media. $\mathbf{D L}_{50}$ : Dosis letal media. ns: No significativo. ${ }^{*}$ : significativo $(\mathrm{P}<0.05) .{ }^{* *}$ : altamente significativo $(\mathrm{P}<0.01)$.

Con el extracto de A. indica se registraron las mayores tasas de eclosión de huevos, lo que indica que tuvo poco efecto en la inhibición de la eclosión de huevos en comparación con el antihelmíntico. Para reducir el 50\% de la eclosión, se requirió una alta concentración $\left(14 \mathrm{mg} / \mathrm{mL}^{-1}\right)$, mientras que los extractos de $\mathrm{P}$. auritum y P. alliacea tuvieron dosis un poco menores $\left(8.7\right.$ y $\left.6.2 \mathrm{mg} / \mathrm{mL}^{-1}\right)($ tabla 2$)$. En otros estudios anteriores (Del moral, 1994; DevonRonald et al., 2012), se ha indicado que el extracto de las hojas del neem actúa contra los adultos y en menor medida contra los huevos y estadios larvarios.

Con una $\mathrm{DL}_{50}$ de $6.17 \mathrm{mg} / \mathrm{mL}^{-1}$, el extracto de P. alliacea fue el producto que tuvo la menor DL. A pesar de esto, los resultados fueron superiores al valor determinado para el antihelmíntico. Las cifras en la literatura indican que esta planta contiene trisulfuros, aceites esenciales, triterpenos, esteroides, alcaloides, flavonoides, taninos, cumarinas, alantonina y ácido oleico, linolénico y palmítico (Pérez et al., 2006), lo que ofrece la posibilidad de uti- 
lizarse como fuente de principios activos con acción antihelmíntica cuando los desparasitantes poseen alta resistencia.

La eclosión de huevos con el extracto de P. auritum también presentó efecto dosis-dependiente, ya que la ordenada al origen $\left(b_{0}\right)$ y la pendiente de regresión $\left(b_{1}\right)$ fueron significativas $(\mathrm{P}<0.05)$. La $\mathrm{DL}_{50}$ observada fue de $8.72 \mathrm{mg} / \mathrm{mL}^{-1}$ semejante a la del extracto de $\mathrm{P}$. alliacea (tabla 2), ambos con un valor mayor a la del antihelmíntico por lo que su actividad antiparasitaria y efecto ovicida no fue tan alto. En la literatura se han descrito diferentes especies de Piper que han mostrado actividad antiparasitaria, entre ellas: P. aduncum frente a Leishmania amazonensis (Caio et al., 1993); y $P$. hostmannianum frente a Plasmodium falciparum (Portet et al., 2007). En años recientes se ha incrementado el uso de aceites esenciales de P. auritum en la búsqueda de nuevos tratamientos antiparasitarios (Antony et al., 2005). En este sentido Camila et al. (2012) reportan una actividad ovicida e inhibición del desarrollo de larvas del extracto de P. tuberculatum en pruebas in vitro.

El extracto de A. indica no afectó en gran medida la eclosión larval y su $\mathrm{DL}_{50}$ fue de $14 \mathrm{mg}$ / $\mathrm{mL}^{-1}$. Esto es que se requiere de una gran cantidad de producto para matar al 50\% de los huevos. Quizá este resultado se debió a que el efecto del neem es acumulativo, tal como lo indica Del Moral (1994), quién manifiesta que no actúa como ovicida, sino que interfiere en los estadios de desarrollo, alterando los procesos metabólicos y de crecimiento de las larvas; además de que afecta la capacidad de fecundación de las hembras.

Hasta ahora se han encontrado fuertes variaciones en la actividad antihelmíntica de los extractos de plantas ricas en taninos y otros metabolitos secundarios, lo cual se debe, posiblemente, a las variaciones en la metodología para su preparación a la naturaleza del extracto; e incluso a la variación en el contenido de metabolitos en las plantas cosechadas en la misma época y también en zonas cercanas, así como las diferencias en el estado fisiológico y edad de las plantas (Alonso-Díaz et al., 2010). Estos factores pueden influir seriamente en el modo o mecanismo de acción dada las características químicas de los metabolitos. Además, una vez que se tiene un extracto, sus características físicas no siempre favorecen cualquier forma de utilización.

Existen numerosos reportes acerca de la eficacia de las plantas ricas en taninos como antihelmínticos en contra de los nematodos gastrointestinales (NGI) de los pequeños rumiantes, tanto de clima templado (Hoste el at., 2006) como en clima tropical (AlonsoDíaz et al., 2010). Sin embargo, los resultados obtenidos a la fecha han sido variables y ninguno aún establece el mecanismo de acción preciso sobre los NGI (Athanasiadou et al., 
2007). Evidentemente esto implica que el uso de extractos todavía requiere de amplio estudio para obtener resultados alentadores.

\section{Conclusiones}

Que los extracto hidroalcohólicos de L. diversifolia, D. tortuosum, L. leucocephala y A. indica presentaron un grado de efectividad contra larvas de nematodos gastrointestinales similares a levamisol.
Que el extracto de L. leucocephala tuvo la menor DL50 de $0.045 \mathrm{mg} / \mathrm{mL}-1$ contra larvas de nematodos gastrointestinales

Que el efecto larvicida de R. mangle y E. cyclocarpum fue mayor al del levamisol al cual los nematodos presentan resistencia.

Que la mayor inhibición de la eclosión de huevos se obtuvo con los extractos de P. alliacea y P. auritum, ya que, con dosis de 6 a 9mg/ mL-1 inhiben el 50\% de la eclosión larval.

\section{Referencias}

Aleman, Y. et al. (2011). Actividad larvicida de extractos de Rhizphora mangle L. contra estrongílidos gastrointestinales de ovinos. Rev. Salud Anim. 33(2): 111-115.

Alonso-Díaz, M.A. et al. (2010). Tannins in tropical tree fodders to small ruminants: A friendly foe? Small Ruminant Research. 89(2-3): 164-173.

Álvarez, A. et al. (1989). Medicina Tradicional y Acupuntura, su aplicación en Veterinaria (SCAMV). La Habana. Ediciones ENPES. Pp. 93-120.

Antony, J.P. et al. (2005). Plant active components -a resource for antiparasitic agents?. Trends in Parasitology. 21(10): 462-8.

Arece, J. et al. (2012). Efecto in vitro del extracto acuoso de Dichrostachys cinerea (L.) Wight y Arn. en el desarrollo de las fases exógenas de estrongílidos gastrointestinales de ovinos. Pastos y Forrajes, 35(3): 301-309.

Athanasiadou, S. et al. (2007). Medicinal plants for helminth parasite control: facts and fiction. Animal. 1(9): 1392-1400.

Athanasiadou, S. et al. (2001). Direct anthelmintic effects of condensed tannins towards different gastrointestinal nematodes of sheep: in vitro and in vivo studies. Veterinary Parasitology. 99(3): 205-19. 
Athanasiadou, S., y Kyriazakis, I. (2004). Plant secondary metabolites: antiparasitic effects and their role in ruminant production systems. Proc Nutr Soc. 63(4):631-639.

Brunet, S. et al. (2008). Effect of the consumption of Lysiloma latisiliquum on the larval establishment of gastrointestinal nematodes in goats. Veterinary Parasitology. 157(1-2): 81-88.

Caio T.S.E. et al. (1999). Selective effect of 2',6'-dihydroxy-4'methoxychalcone isolated from Piper aduncum on Leishmania amazonensis. Antimicrob Agents Chemother. 43: 1234-41.

Calderon-Quintal, J.A. et al. (2010). Adaptation of Haemonchus contortus to condensed tannins: can it be possible?. Med. Vet. 43(3): 165-171.

Camila O. et al. (2012). The anthelmintic effect of plant extracts on Haemonchus contortus and Strongyloides venezuelensis. Veterinary Parasitology. 183(3-4): 260-268.

Del Moral, M. (1994). Utilización del extracto acuoso del árbol Neem en el control de helmintos gastrointestinales en becerros. Trabajo especial de Grado. Universidad Nacional Experimental Francisco de Miranda, Ciencias del Agro y del mar. Coro, Venezuela. p. 51.

Devon-Ronald, D. et al. (2012). Eficacia del extracto de las hojas del Neem Azadirachta indica A. Juss en el control de nematodos gastrointestinales en ovino Pelibuey. REDVET. 13(7).

Florence, K.T., y Mbida, M. (2011). In vitro activities of acetonic extracts from leaves of three forage legumes (Calliandra calotyrsus, Gliricidia sepium and Leucaena diversifolia) on Haemonchus contortus. Asian Pacific journal of tropical medicine, 4(2): 125-128.

García E. (1988). Modificaciones al Sistema de Clasificación Climática de Köppen. 4a ed. Editorial Instituto de Geografía. Universidad Nacional Autónoma de México, México, Pp

Githiori, J.B. et al. (2006). Use of plants in novel approaches for control of gastrointestinal helminthes in livestock with emphasis on small ruminants. Veterinary Parasitology. 139(4): 308-320.

González-Garduño, R. et al. (2010). Efecto del hipoclorito de sodio y extracto de cítricos en la reducción de la infestación con nematodos gastrointestinales resistentes a antihelmínticos en ovinos de pelo. Rev Mex Cienc Pecu. 1(2): 179-187. 
González-Garduño, R. et al. (2014). Diagnóstico in vitro y en campo de resistencia antihelmíntica en nematodos gastrointestinales de pequeños rumiantes. Arch Med Vet. 46: 399-405.

Hoste, H. et al. (2006). The effects of tannin-rich plants on parasitic nematodes in ruminants. Trends in Parasitology. 22(69): 253-261.

Lanusse, C., y Prichard, R. (1993). Relationship between pharmacological properties and clinical efficacy of rumiants anthelmintics. Veterinary Parasitology. 49(2-4): 123-158.

Makkar, H.P.S. et al. (2007). Bioactivity of phytochemicals in some lesser-known plants and their effects and potencial applications in livestock and aquaculture production systems. Animal. 1(9): 1371-1391.

Marie-Magdeleine, C. et al. (2010). In vitro effects of Tabernaemontana citrifolia extracts on Haemonchus contortus. Research in Veterinary Science. 89(1): 88-92.

Marie-Magdeleine, C. et al. (2009). In vitro effects of Cucurbita moschata seed extracts on Haemonchus contortus. Veterinary parasitology, 161(1): 99-105.

Márquez-Lara, D. (2003). Resistencia a los antihelmínticos: origen, desarrollo y control. Revista Corpoica. 4(1): 55-71.

Marrero, E. et al. (1994). Actividad antihelmíntica de Bromelia pinguin L (Bromeliaceae) en terneros. Rev Salud Anim, 16(1-3): 63-68.

Olivares, J. (2001). Oesophagostomum columbianum: Puesta en evidencia, caracterización y control en ovinos de la región de Huichapán, Estado de Hidalgo, México. Tesis presentada en opción al grado de Doctor en Ciencias Veterinarias. CENSA. La Habana, Cuba. 137 p.

Papadopoulos E. (2008). Anthelmintic resistance in sheep nematodes. Small Rumin Res. 76: 99-103.

Pérez, R. et al. (2006). Actividad citotóxica y antioxidante de Petiberia alliacea L., Revista Chapingo serie Horticultura. 12(1):51-56.

Pietrosemoli, S. et al. (1999). Empleo de hojas de Neem (Azadirachta indica A. Juss) en el control de nematodos gastrointestinales de bovinos en pastoreo. Rev. Fac. Agron. (LUZ). 16(1): 220-225. 
Quesenberry, K.H., y Dunn, R.A. (1987). Variability among Desmodium species for response to root-knot nematodes. Crop science, 27(6): 1234-1237.

Reed, J.D. (1995). Nutritional toxicology of tannins and related polyphenols in forage legumes. J. Anim. Sci. 73(5): 1516-1528.

Salazar, E. (2004). Endoparasite control with Nim (Azadirachta) International Conference on Goat. South Africa, 4 -9 July, 2004. Book of Abstracts: 79.

SAS Institute. (1999). The SAS System for Windows. Version 8. SAS Institute. Inc. Cary, N. C. USA.

Shalaby, H.A. (2013). Anthelmintics Resistance; How to Overcome it?. Iranian journal of parasitology, 8(1), 18.

Taylor, M.A. et al. (2009). Multiple resistance to anthelmintics in sheep nematodes and comparison of methods used for their detection. Small Ruminant Research. 86: 67-70.

Thienpont D. et al. (1986). Diagnóstico de las helmintiasis por medio del examen coprológico. Janssen Research Foundation, Beerse, Bélgica, Pp. 19-43.

Torres-Acosta, J.F.J. et al. (2012). Nutritional manipulation of sheep and goats for the control of gastrointestinal nematodes under hot humid and subhumid tropical conditions. Small Ruminant Research. 103(1): 28-40.

Wagland, B.M. et al. (1992). A new simplified assay for larval migration inhibition. Int J Parasitol. 22(8): 1183-1185. 